(C) [2009] IEEE. Reprinted, with permission, from Li, Zheng and Hoang, Doan 2009, 'Overlapping Impacts and Resource Coodination for High-density Wireless Communication', 2009 IEEE-RIVF International Conference on Computing and Communication Technologies, pp. 180-186. This material is posted here with permission of the IEEE. Such permission of the IEEE does not in any way imply IEEE endorsement of any of the University of Technology, Sydney's products or services. Internal or personal use of this material is permitted. However, permission to reprint/republish this material for advertising or promotional purposes or for creating new collective works for resale or redistribution must be obtained from the IEEE by writing to pubs-permissions@ieee.org. By choosing to view this document, you agree to all provisions of the copyright laws protecting it. 


\title{
Overlapping Impacts and Resource Coordination for High-density Wireless Communication
}

\author{
Li Zheng and Doan B. Hoang \\ iNEXT - UTS Centre for Innovation in IT Services and Applications, Faculty of Information Technology, \\ University of Technology, Sydney, NSW 2007, Australia
}

\begin{abstract}
A high-density wireless environment could not avoid cell/BSS (Basic Service Set) overlapping, which may cause Co-channel interference. Such interference makes it difficult to support QoS. It is desirable that BSSs can share a single frequency channel without interference and at the same time, QoS is supported. Additional functionalities particularly in MAC layer are required to achieve interference-free for a businesssound wireless deployment. In this paper, we present a detailed analysis on OBSS interference, caused by overlapping, in relation to MAC functions and propose a resources coordination scheme to support OBSS co-existence and QoS, based on the context of 802.11 WiFi as an example. The uplink and downlink transmission are separated into two periods. The grouping assignments using coloring theory are carried out based on the topology to divide BSSs into groups and time span to avoid downlink collision.
\end{abstract}

Keywords - BSS overlapping, interference, Quality of Services, Resources coordination, WLAN, coloring theory for group assignment, and multimedia service differentiation.

\section{INTRODUCTION}

Both WiFi and WiMAX have been rapidly deployed with telecommunication operators, particularly in a high density environment, such as airport, university campus and office building, which are normally equipped with large number of BSSs. As these BSSs are densely located within a small area, their transmission ranges may overlap and this overlapping causes interference among each other. The term Overlapping Basic Service Sets (OBSS) is used to describe this service environment. Due to the limited availability of channels implies that they must be re-used, much like in cellular networks. In order to achieve interference-free in the shared environment, an in-depth analysis would be studied in dimension of location distance, frequency distance, and Medium Assess Control (MAC) function.

Based on those analyses, certain coexistence/ coordination on the MAC protocol would be introduced for these multiplecell WLAN, to allocate resources among BSSs in order to reuse frequency channels while avoiding co-channel interference between BSSs. In [1], Mangold proposes game theory to allocate resources among overlapping WLAN and Bluetooth, with assumption of absence of central coordination. In [3] and [4], similar coexistence mechanisms based on Clear Channel Assessment and traffic scheduling techniques, are introduced to mitigate interference between IEEE 802.11 WLANs and Bluetooth-based WPANs. Centralized Control is introduced in [6] and [5], where a 'Super Point Coordination' is proposed to work out possible collision sets, which is only useful in cases where there is only a small number of connections or in small networks. All of theses studies either focus on coexistence between two standards when there is no central coordination, or lack of scalability of the problem even when central coordination is proposed. In this paper, we emphasize that the overlapping avoidance is insufficient in a high-density scenario, and propose an overlapping coordination to support Service Differentiation, which focuses on solving interference problem among a high density WLAN domain where central resources management is already in place in a Hybrid framework [7]. The re-use strategies used improve channel utilization, while our grouping algorithm provides scalability, comparatively.

\section{OVERLAPPING IMPACTS ANALYSIS}

Generally OBSS is referred when two or more BSSs running at the same channel, where the transmissions by some clients belonging to one BSS affect stations (clients) in the other BSSs. Regarding performance under OBSS, [1][2] shows that a wide range of delays and throughputs degrades are observed, depending on the degree of overlapping and the number of overlapped BSSs. And such delay and throughput reduction could not be forecasted and is difficult to control. Below, we focus on how the degrades / collisions are clearly caused by OBSS effects.

\section{A. Inter-BSS Distance Effect}

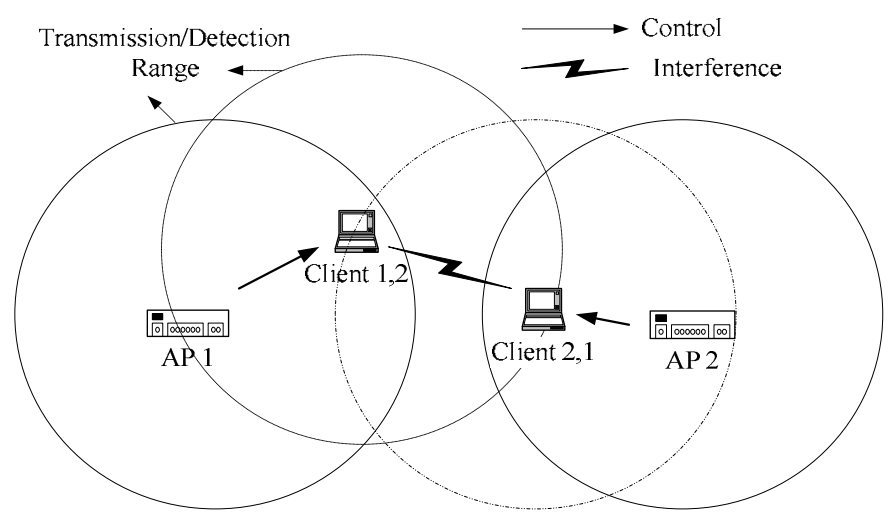

Figure 1 Non-AP-Overlapped: client $_{2,1}$ is not reachable from $\mathrm{AP}_{1}$.

It is commonly known that location distance and frequency distance are major factors in determining the levels of interference. Figure 1 shows an example of this problematic situation, since client 2,1 , belonging to $\mathrm{AP}_{2}$, could hear from the 
client $t_{1,2}$ of $\mathrm{AP}_{1 . \text {, }}$ it could not receive beacons transmitted by $\mathrm{AP}_{1}$, and hence it would not set up its NAV during the CFP (Contention Free Period) of BSS $_{1}$. Therefore, client 2,1 may transmit a frame while client ${ }_{1,2}$ is receiving a data frame from $\mathrm{AP}_{1}$ during a CFP, resulting in a collision. Obviously, the closer the distance of two BSSs is, the higher potential of the interference would be and the worse interference would be. The example in Figure 2 shows such situation, where two APs move closer to each other, client ${ }_{2,1}$ does not initiate a data transmission during the CFP of $\mathrm{BSS}_{1}$ after hearing the beacon frame from $\mathrm{AP}_{1}$ at the beginning of a CFP. This leads to large delay and reduced throughput in client ${ }_{2,1}$ additionally, apart from the common co-channel interference between client ${ }_{2,1}$ and client $t_{1,2}$ as discussed above.

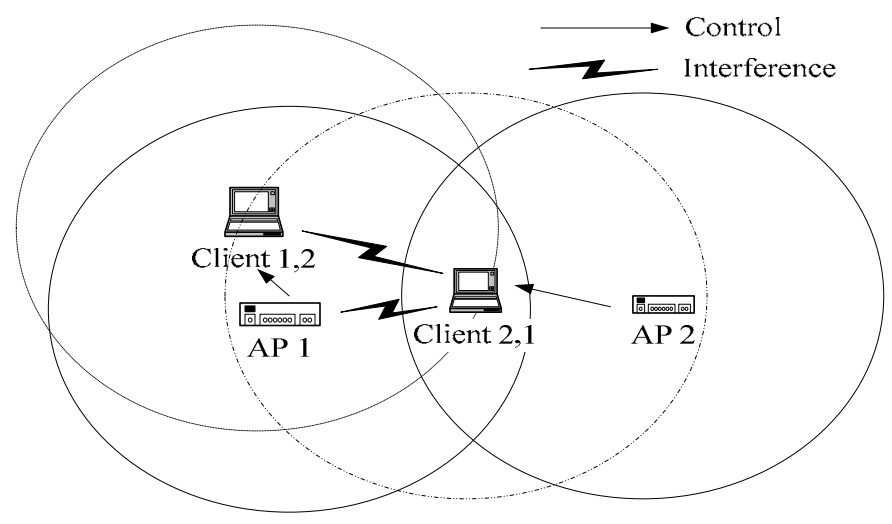

Figure 2 AP-overlapped Scenario: client ${ }_{2,1}$ is reachable from $\mathrm{AP}_{1}$

\section{B. Overlapping Effect on Medium Access Scheme}

Besides distance impact, the medium access schemes operating in the interfering parties could also make an impact. At first, we look at the scenario if both two parties adopt contention scheme when they overlaps each other. The fundamental of contention scheme is Carrier Sense Multiple Assess /Collision Avoidance (CSMA/CA). Both DCF and EDCF define a Collision Avoidance mechanism to reduce the probability of collision. Therefore, even two BSSs overlap each other, station could still use such Collision Avoidance mechanism to avoid any potential collision, not only with stations in its own BSS, but also with stations in other BSS, which it could detect. On the other hand, hidden terminal problem becomes worse as the entire system serves more stations and covers wider areas. However, the medium could be still fully utilized in such a random access scheme while collision rate is acceptable.

In the case that both parties adopt the polling scheme, the interference effect may be even worse. The main reason is that when the polling scheme operates without the CSMA/CA contention window randomization and backoff operations in the contention scheme, there is a risk of repeated collisions if multiple, overlapping, point-coordinated BSSs are operating on the same PHY channel, and their CFP rates and beacon intervals are approximately equal. Figure 3 shows the beginning of collision of two CFPs in such situation. In IEEE802.11 standards, to eliminate such problem, a random backoff delay and a DIFS are suggested to start a CFP when the initial beacon is delayed because of deferral due to a busy medium. However, this is insufficient to eliminate all possible interferences, because both APs may not hear from each other. Even two APs could hear from each other, the medium could also be grabbed by a foreign station, which is hidden and performs additional backoff and DIFS. In the case of these repeated collisions, polling transmissions could not provide guaranteed service at all, which is supposed to be promised in polling scheme as its design objective. OBSS becomes one of critical challenges for polling scheme.

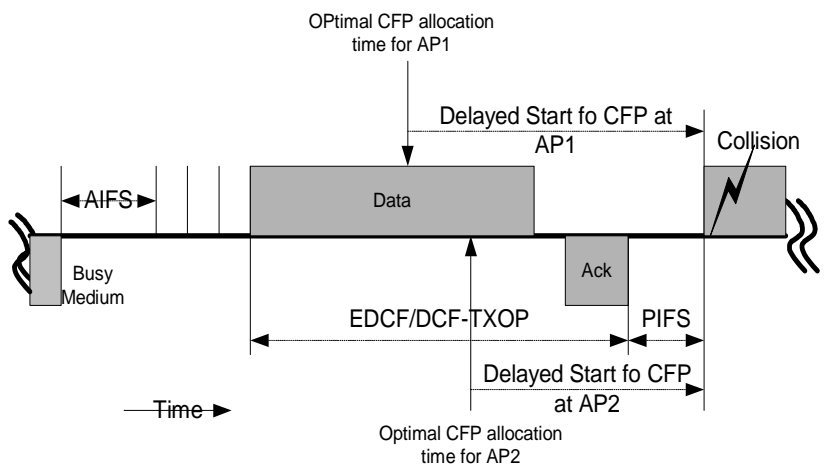

Figure 3 Frame Collisions of Two Delayed CFPs

In the case of combination of polling scheme and contention scheme for two parties, which overlap each other, interference becomes complex. Note that polling scheme always has priority over contention scheme. Therefore, BSS, which operates on polling scheme, may capture the medium over another BSS, which operates on contention scheme. And such capture may not be fair to the BSS, operating on contention scheme, even though collision may not be serious. Furthermore, in the case of non-AP-overlapped scenario, all stations may not be in the detection range of a foreign AP, which operates on polling scheme. Those stations, which are out of range of detection of such AP, become hidden to this AP. They perform channel contention regardless of polling period in other BSS and cause serious collision. In summary, QoS supports become problematic when BSSs overlap, regardless of how access schemes are used when there is no co-ordination in place among BSSs. It is only a matter of interference severity in relation to different combinations of MAC schemes among BSSs.

\section{Neighborhood Capture Effect}

The neighborhood capture was firstly pointed out in [6]. Its effect can be understood by considering three BSSs locate closely and are assigned the same frequency channel. Besides that they experience the normal co-channel interference, 
medium could be captured unfairly by certain BSSs. As illustrated in Figure 4 each BSS could only detect the transmission of its respective neighbor BSS. So BSS 1 and BSS 3 are hidden to each other and they operate independently at the same time. While all stations in three BSSs use CSMA protocol to access medium, a station or BSS may refrain from transmitting while it detects the channel busy. Once a station in BSS 1 starts a frame exchange, stations in BSS 2 would defer transmission as they could detect the current BSS1 transmission. However, it implies that the stations in BSS 3, which is hidden to BSS 1 and do not detect the BSS 1 transmission, could start its frame exchange independently during the BSS 1 transmission. This could be repeated as long as BSS 1 and BSS 3 have data to transmit. As a result, stations in BSS2 may have no opportunity for transmission for long period, while the medium is captured by the neighborhood of BSS 2. This leads to large delay and reduced throughput in BSS 2 with such unfair treatment.

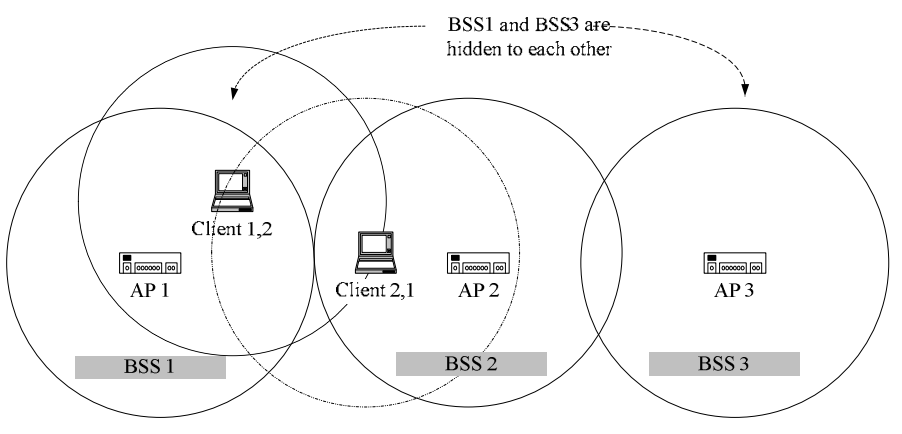

Figure 4 Neighbourhood Capture

\section{RESOURCES COORDINATION IN A OVERPLAYING ENVIRONMENTS}

The analysis above actually propels us to propose a WLAN MAC resource coordination scheme for allocating resource among overlapping BSSs by re-using the frequency channel efficiently while avoiding co-channel interference.

\section{A. Coordinated and Synchronized Superframe}

In an OBSS environment, the contention scheme appears to be a better option than polling scheme in combating interferences. However, unfairness issue between uplink and downlink in the contention scheme is still a major problem. In contrast, with the polling scheme, the downlink traffic is piggybacked on the poll frame for broadcast while the uplink traffic is transmitted after poll frame by individual stations. Therefore, uplink and downlink traffic are generally given equal opportunities in polling scheme. In order to maximize the benefits of using contention scheme in OBSS environment, unfairness issue between uplink and downlink has to be solved. One option is to separate uplink and downlink transmission while allocating separate resources dynamically and individually for downlink and uplink transmission. Such treatment should be used to guarantee the equal opportunity for downlink.

Therefore, the OBSS-coordinated medium access alternates between thee Downlink Polling Access and Uplink Contention Access. All BSSs in such coordinated OBSS environment would be synchronized and have a unique and coordinated superframe structure. The downlink period alternates with an uplink period. Each coordinated BSS simultaneously operates in either downlink period for polling access or uplink period for contention access only. The resource manager (RM) generates a downlink period at the downlink repetition rate, which is also called Superframe size. The Superframe length (downlink period repetition rate) is a multiple of DTIM (DTIM is a basic time unit defined in the IEEE 8021.11); but it should not be constrained to a certain multiple of DTIM and dynamically controlled by the Resource Manager according to the traffic demands. A common example of the superframe is illustrated in figure 5, where a beacon interval is a DTIM. The superframe consists of five DTIM and each time span is a DTIM period for each re-use group of total three. It is not allowed to have delayed beacon and foreshortened downlink period by using strict synchronization within the entire OBSS environment. The beacon frames are regularly broadcasted in every beacon interval from AP stations to all client stations. The Beacon frame contains necessary information for synchronization and contention procedure, including the time length of uplink/downlink period and QoS service differentiations.

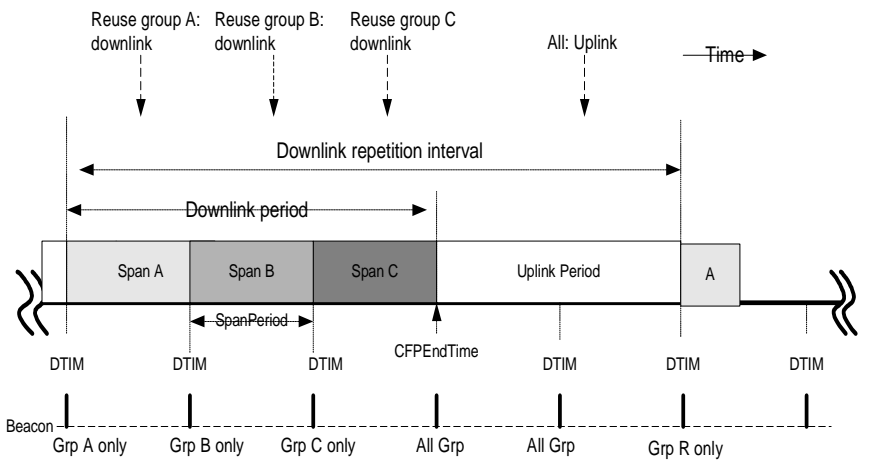

Figure 5 An Example Superframe

\section{B. Uplink Transmission in a 'Super Cell'}

For uplink transmission in OBSS, contention-based scheme is proposed for all BSSs. As discussed earlier, if all BSSs operate in contention scheme, the co-channel interference is minimum as the collision avoidance mechanism is used. Within a finite relevant detention range the medium is fully utilized for contention-based access. Once the stations are out of the detention of the station, which is currently occupying the medium, they are free to contend for the medium without any further coordination during the uplink period. During the uplink period, the entire OBSS is 
considered a 'super cell' on a larger scope, operating in contention-based scheme. Contention-based scheme also has a number of desirable properties: simplicity, self-organization and efficiency.

During uplink period, all client stations in all BSSs become active for uplink transmission. And beacon frames are regularly broadcasted in every beacon interval from AP stations to all client stations. The Beacon frame contains necessary information for synchronization and contention procedure, including the time length of uplink period and QoS service differentiations. The uplink access method in a coordinated OBSS environment could be an EDCF in which priority-based QoS mechanism is supported while collision avoidance is still remained. The details of EDCF access procedures are given in IEEE 802.11e specifications and other published articles, while we only address the concerns on the unique QoS provisioning (traffic class mapping) among each participating BSS in order to provide fair service differentiation as detailed below.

The QoS facility of EDCF supports 8 user priorities, which are identical to the IEEE $802.1 \mathrm{D}$ priority tags. One or more user priorities shall be assigned to one of four EDCF access categories (AC). The QoS mapping table is the guidelines for mapping traffic types to different EDCF priorities. The admitted TS (such as VoIP and video) should be assigned higher ACs to meet the delay requirements. Each AC is an enhanced variant of the DCF that contends for TXOPs using one set of EDCF QoS parameters element in Beacon frames. According to the principle of fairness among BSSs, the access to the QoS service differentiation should be equal and fair among BSSs. All the clients are treated 'equally' in the entire OBSS system according to the EDCF access rules. Therefore, implementation values for EDCF QoS parameters set and the mapping above between user priorities and AC should be unique to all BSSs and controlled by the RM. In such way, all BSSs are guaranteed equal for accessing prioritized treatments for delay-sensitive / critical message and data.

\section{The Downlink Transmission with BSS grouping}

For downlink transmission in OBSS, polling scheme is used to provide transmission opportunity. During downlink period, as polling scheme is used, AP could only poll itself for all downlink traffic while all uplink traffic are scheduled during the uplink period. However, interference is obvious if polling schemes are used among OBSS without coordination as discussed. Therefore, channel assignment, as the key part of the downlink coordination, is essential in order to avoid OBSS interference and maximize resources utilization. The scarcity of spectrum necessitates efficient channel assignment mechanisms. Whether the channel sharing is based on upon time (Time Division Multiple Access), frequency (Frequency Division Multiple Access), code (Code Division Multiple Access), or a combination thereof, there exists the fundamental limit on the number of users sharing the same channel assignment simultaneously.

This has motivated the need for spatial reuse of the channel. In our OBSS downlink channel assignment solution, the channel sharing is based upon time, spatial reuse is to have users (BSSs) sufficiently far apart (in a reuse group) use the same frequency band. Members in the same reuse group are generally interference-free to each other and have certain physical distance sufficiently far apart from each other. Neighboring BSSs, which are in different groups, could not be assigned the same channel simultaneously. Therefore, the downlink period is divided into several limited time slot/span for these reuse groups. Apparently, the less number of time slots are divided, the more efficient the channel assignment solution is. We apply graph coloring technique to solve this grouping problem with details in Sub-Section D.

The downlink period itself consists of several time spans for different reuse groups of BSS. Each re-use group is at least assigned one individual time span. The reuse group is defined as a collection of BSSs, such that any two of BSSs in the group would not overlap each other. When a certain reuse group is assigned active during an assigned time span, its member BSSs are allowed to be active at that time span. Each downlink time span has equal/approximately equal length according to the fairness principle among each reuse group.

The AP stations then start transmission with the Beacon frames. The beacon frame would firstly set NAV (Virtual carrier sense) in all client stations in this active BSS and also carry the necessary information for polling period, such as polling order, and polling duration. Other groups should just remain silent by setting their NAV until they are assigned active. They should not even transmit Beacon frame during their silent periods to avoid any possible Beacon collision with the current active group. Each AP also has full controls and options for varied service differentiation for its own traffic only during its assigned downlink polling transmission period. The adoption of different service differentiation schemes in each BSS would not make any effect to transmissions in its neighboring BSSs. The AP is equipped with a QoS scheduler and should always satisfy (offer certain TXOPs) the admitted TS under the controlled polling mechanism based on the accepted TSPEC information of the admitted TS.

\section{First Grouping and Second Grouping}

The number of time span (re-use group) is calculated in $\mathrm{RM}$ using certain grouping (first and second) algorithm. The purpose of channel (group) assignment computation is to produce guidelines for dividing all participating BSSs into several re-use groups, namely Group R (Red), B (Blue), G (Green), Y (Yellow), etc. The constraint of grouping is that no two overlapping BSSs could be assigned into the same group. The objective of grouping is to find the minimum number of 
groups for complete coverage. The decision purely depends on the number of BSSs in the coordinated environment and the overlapping severity.

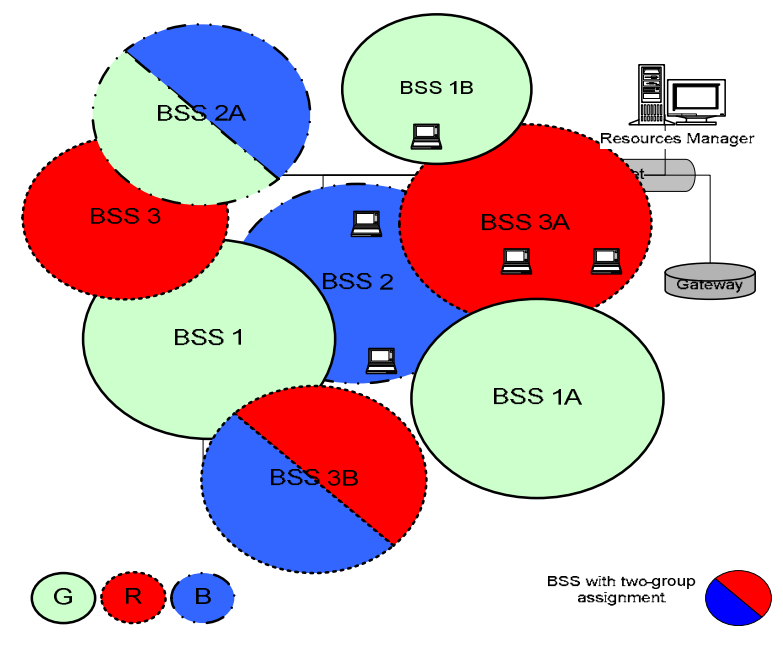

Figure 6 An Example of Second Group Assignment

BSSs with high traffic demands may deserve long period for transmission when possible. However, due to the need of synchronization, equal-length span would be only considered. Therefore, uneven span is inappropriate and additional second time span is proposed. In order to maximize the spatial reuse, we propose second grouping assignment, in which BSSs with high downlink traffic may be additionally assigned its second group to satisfy their demands. The second grouping neither increases the number of groups used nor changes the result of the first grouping assignment. Instead, it only assigns additional group to certain BSSs with high traffic demands. The constraint of the second grouping remains the same as that in the first grouping. Figure 6 shows the results of the first and second grouping for an 8 co-located BSSs environment. All BSSs are assigned into 3 groups of R (BSS 3, 3A, 3B), B $(2,2 \mathrm{~A})$ and $\mathrm{G}(1,1 \mathrm{~A}, 1 \mathrm{~B})$; while BSS $2 \mathrm{~A}$ and $3 \mathrm{~B}$ are assigned with two groups. Obviously, each BSS is at least assigned one group and such group contains BSSs members, which do not overlap and interfere with each other. The detailed grouping algorithm using coloring theory would be given in [8].

\section{PERFORMANCE SUMmary AND SimUlation RESUltS}

The performance should be examined in terms of QoS, medium utility and fairness among the participated BSSs. This section presents these performance results of our proposed scheme. The values of simulation attributes used are standard to WLAN IEEE 802.11 in OPNET environment. The network configuration in the downlink simulation consists of the three groups with their representatives, BSS 1, 2, and 3 (as in Figure 6), which co-locate and overlap with each other. Each BSS would only have downlink traffic and its AP simultaneously transmits multiple applications (VoIP, light Video Conference (VC), Audio and bulk data) to its clients at all time. Each BSS is loaded with different traffic. BSS 1 has three VoIP transmissions; BSS 2 houses light VC and Audio; and BSS 3 is overloaded with large bulk data.

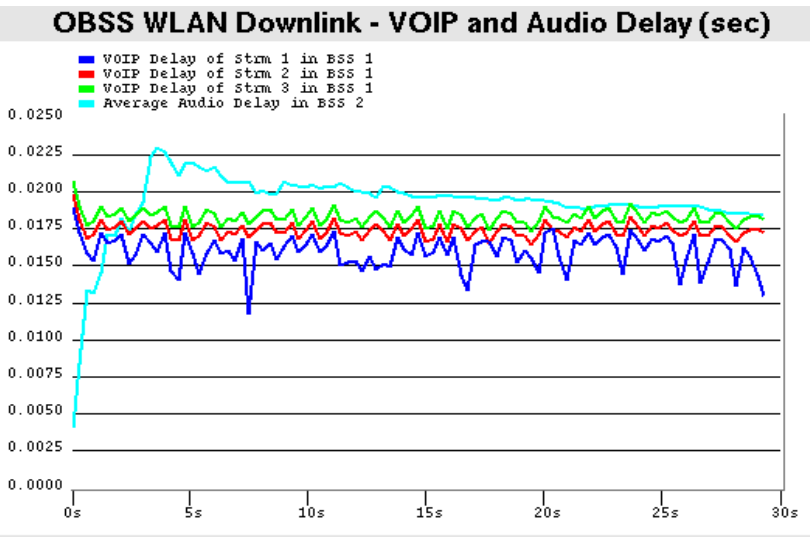

Figure 7 VoIP and Audio Delay in BSS 1and 2 of Downlink Scenario

As seen in Figures 7, three VoIPs in BSS 1 have good delay performances, which all are less than 20ms. BSS 2 also maintains a satisfied performance for an audio and light VC, as its audio stream performs at less than 25ms delay (Figure 7) and light VC achieves approximately 600Kbps (Figure 8). As seen, service differentiation is provided. However, bulk data in BSS 3 experiences a data drop at $1.75 \mathrm{Mbps}$ as shown in Figure 8 . The data application reaches the capacity of BSS 3 during the assigned duration and achieved a throughput of $1.8 \mathrm{Mbps}$ only. While BSS 3 is overloaded with such aggressive bulk data and has consequent data drop, it could not gain further transmission opportunities and make any impact on other applications from the co-located BSS 1 and 2. The results demonstrate fairness among the co-located BSSs.

The uplink network configuration is as the same as in the downlink except that there are only Uplink traffic rather than downlink. Additionally, in order to outline the impact of SD (Service Differentiation), the simulations are designed both with SD mechanism and without SD mechanism. As seen in Figure 9, delays of all VoIP applications in SD (Service Differentiation) scenario are guaranteed with the satisfied delay performances, except the one in the scenario without SD. Also, the VoIP streams in different BSSs are all assigned the same AC according to the unique service differentiation assignment principle, so the delay performances of all VoIP are approximately the same. Particularly, the VoIP in BSS 1 and 2 are not affected by the aggressive bulk data application in BSS 3, which is regulated by SD. Therefore, unique service differentiation ensures fairness and the performances of the delay-sensitive applications. 


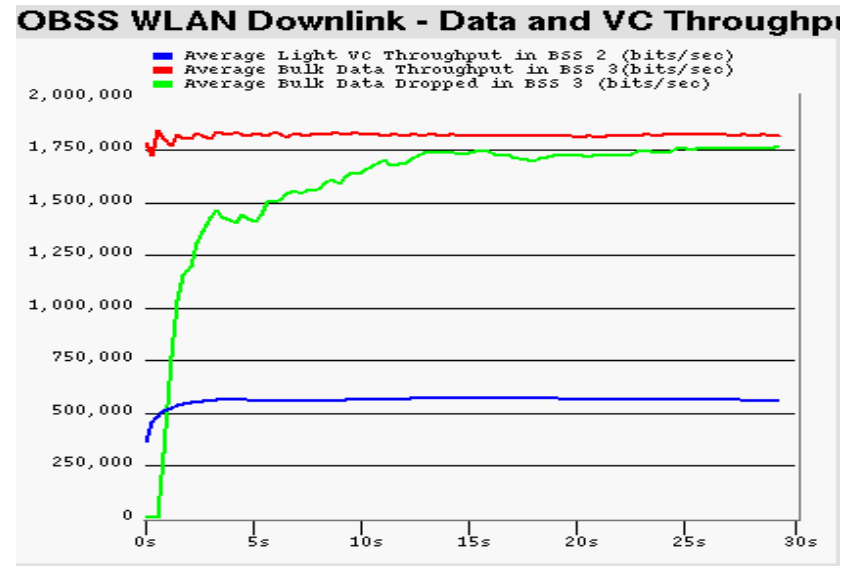

Figure 8 VC and Bulk data Downlink Throughput in BSS 2 and BSS 3

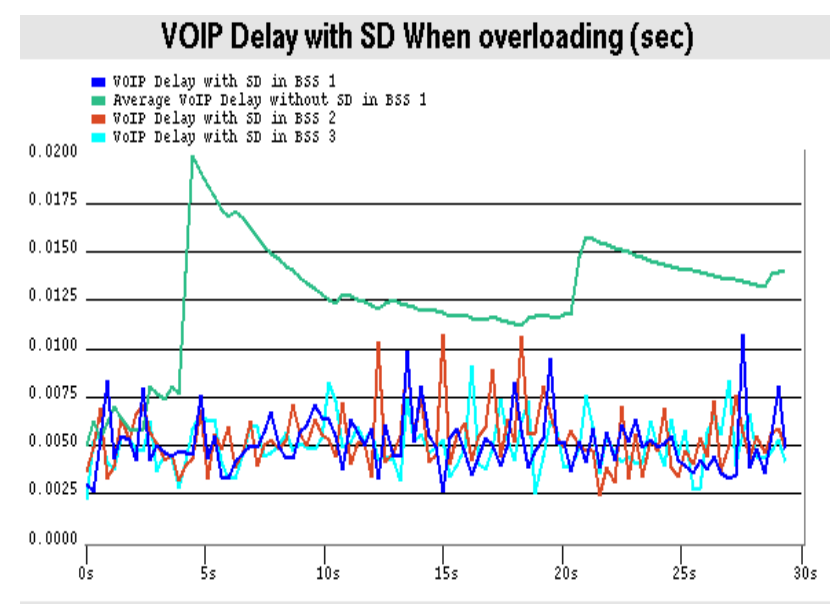

Figure 9 VoIP Delay Performances in Uplink Scenario

The results above assume parameters are set at the optimal values. In fact, parameters, mainly the superframe length, do have great impact on the overall performance. Figures 10 shows the performance variations of VoIP delay (Downlink scenario, in BSS 1) among several environments where different superframe durations are implemented. As seen, the shorter the superframe is, the best VoIP delay performance it could be, as superframe repetition is more often. However, further shortening superframe would not gain any more delay improvement; a lower threshold, to prevent the system degrades on delay, is recommended between 30ms-60ms.
OBSS WLAN Downlink - VoIP Delay Performance (sec)

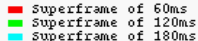

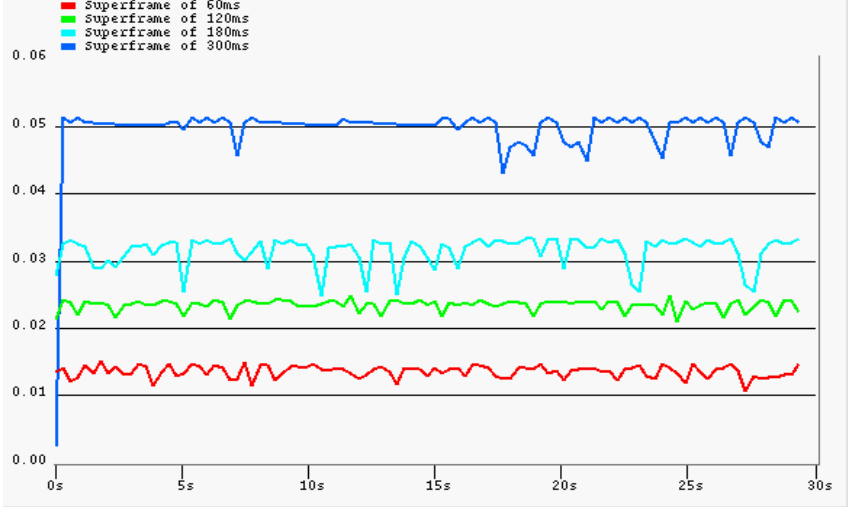

Figure 10 VoIP Delay in Downlink Scenarios with Different Superframe Length

It should be noted that the best delay performance of VoIP could also mean a low throughput in total as a trade-off, particularly for large applications, such as bulk data and Video Conference (VC). When a large Beacon is 324 octets and it is transmitted in a BSS_Basic_Rate (normally 1Mbits/s for management and control frames), it takes approximately $2.4 \mathrm{~ms}$ overhead for its transmission. The overhead for 3-4 beacons may be considered relatively large in a $20 \mathrm{~ms}$ superframe. Therefore, the medium efficiency is better when a longer superframe is used. Figure 11 shows such case when a 20ms superframe is implemented, the full throughput of videoconferencing in BSS 2 could not even be guaranteed. Further experiments suggest an upper threshold of $180 \mathrm{~ms}-120 \mathrm{~ms}$ is sufficient enough to ensure throughput performance for large application. In order to cater both VoIP delay and goodput performance of bulk applications like VC, the optimal value of superframe length is recommended within the range of $60 \mathrm{~ms}$ to $120 \mathrm{~ms}$, based on our generic traffic profile.

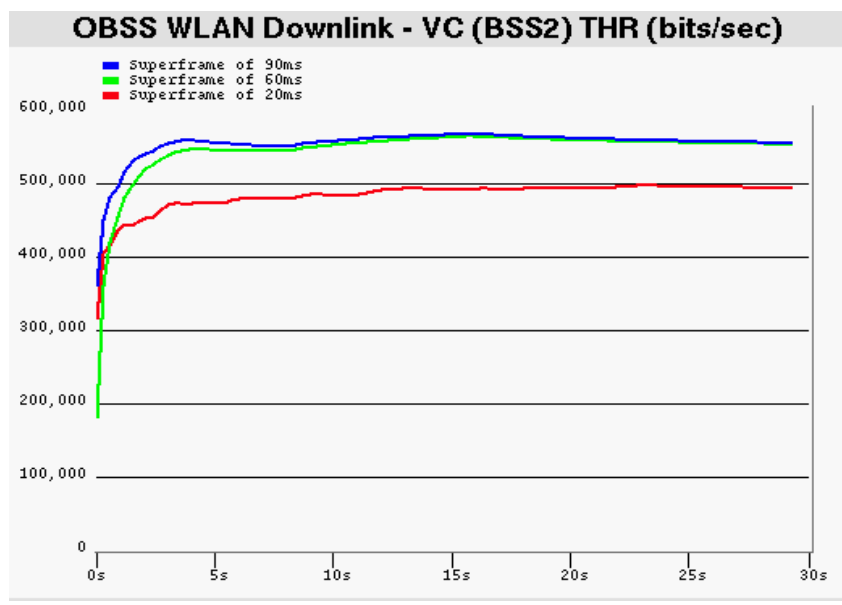

Figure 11 VC Throughputs in Downlink Scenarios, BSS 2 with Different Superframe Length 
OBSS WLAN Downlink - Data Throughptu (bits/sec)

$4,000,000$

E Throughput with one-Group Assignment

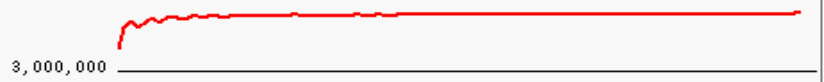

$2,000,000$

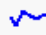

$1,000,000$

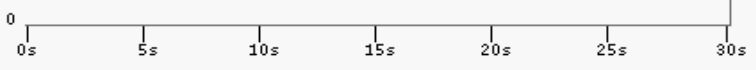

Figure 12 Bulk Data Throughput in Downlink Scenario, BSS 3 with Second Assignment

Finally, Figure 12 shows the efficiency improvement in term of bulk data throughput in BSS 3, gained by using both second \& first grouping assignment via coloring theory, compared to the first grouping assignment scenario. Apparently, such improvement is obvious and the bulk data in BSS 3 is then satisfied with second timeslot allocation. At the same time, the results also show no performance degrade in other applications, such as VC and VoIP, either in the same BSS or in its neighboring BSSs. It proves the second grouping would not affect the first grouping at all, while largely providing medium efficiency.

\section{CONCLUSIONS}

We have observed the unavoidable problem of overlapping BSS and pointed out that a centralized coordination should be in place to solve the OBSS interference problem, particularly in a common high-density environment. The intensive analysis on the OBSS interference has firstly been outlined. Based on this, the coordination is proposed and it separates uplink and downlink transmission into two periods, which are supported by contention and polling mechanisms respectively. The grouping assignments are carried out based on the topology to divide BSSs into groups and time span to avoid downlink collision. Group members are then synchronized for transmission. The experiment results indicated that our resource coordination scheme is not only fair to member BSSs but also effective in avoiding collision and providing QoS service differentiation. Future works of applying such scheme to mobile $3 \mathrm{G} / 4 \mathrm{G}$ networks would be also interesting.

\section{REFERENCES}

[1] S. Mangold, L. Berlemann, B. Walke, "Equilibrium Analysis of Coexisting IEEE 802.11e Wireless LANs", 14th IEEE International Symposium

[2] Dunat, J.-C.; Elicegui, L.; Bonnet, C.; "Impact of inter-cell interference in a IEEE 802.11a network with overlapping cells”, Personal, Indoor and Mobile Radio Communications, 2004.

[3] Chevrollier, N.; Rebala, O.; Golmie, N.; "Bluetooth and WLAN coexistence: challenges and solutions", Wireless Communications, IEEE, Dec. 2003

[4] Golmie, N.; Rebala, O.; "Bluetooth adaptive techniques to mitigate interference”, Global Telecommunications Conference, 2003. GLOBECOM '03 IEEE.

[5] T.M.Eladly, H.A.; Chen, T.M.; "An overlay algorithm to improve the support of multi-hopping in the IEEE 802.11 WLANs", Communications, 2004 IEEE International Conference.

[6] Vergados, D.D. Vergados, D.J., "Synchronization of multiple access points in the IEEE 802.11 point coordination functions", Vehicular Technology Conference, 2004.

[7] Zheng L., Dadej A., Gordon S., "Hybrid Quality of Service Architecture for Wireless/Mobile Environment”, IFIP TC6: Interworking 2002 on Converged Networking Data \& Real-Time Over IP; Oct 02, Australia.

[8] Zheng L. and Hoang D. B., "Applying Graph Colouring in Resource Coordination for a High-density Wireless Environment", IEEE 8th International Conference on Computer and Information Technology, Sydney, 2008. 